Kragujevac Journal of Mathematics

Volume 38(2) (2014), Pages 245-248.

\title{
A NOTE ON GENERALIZED QUASI-BAER RINGS
}

\author{
M. ANZANI ${ }^{1}$ AND H. HAJ SEYYED JAVADI ${ }^{1}$
}

\begin{abstract}
A ring with identity is generalized quasi-Baer if for any ideal $I$ of $R$, the right annihilator of $I^{n}$ is generated by an idempotent for some positive integer $n$, depending on $I$. We study the generalized quasi-Baerness of $R[x ; \sigma ; \delta]$ over a generalized quasi-Baer ring $R$ where $\sigma$ is an automorphism of $R$.
\end{abstract}

\section{INTRODUCTION}

Throughout this paper $R$ denotes an associative ring with identity. Recall that $R$ is (quasi-) Baer if the right annihilator of every (right ideal) nonempty subset of $R$ is generated as a right ideal by an idempotent. It is easy to see that the Baer and quasiBaer properties are left-right symmetric for any ring. In [8] Kaplansky introduced Baer rings to abstract various properties of $A W^{*}$-algebras and von Neumann algebras. Clark defined quasi-Baer rings in [4] and used them to characterize when a finite dimensional algebra with unity over an algebraically closed field is isomorphic to a twisted matrix units semigroup algebra.

As a generalization of Baer rings, $R$ is called a right (left) p.p.- ring if every principal right (left) ideal is projective. Equivalently, if the right (left) annihilator of any element of $R$ is generated by an idempotent of $R$. $R$ is called a p.p.-ring (also called a Ricart ring [10, p. 18]), if it is both right and left p.p.-ring. In [6] Huh et al. defined a ring $R$ to be called generalized right p.p.-ring if for any $x \in R$ the right annihilator of $x^{n}$ is generated by an idempotent for some positive integer $n$. von Neumann regular rings are p.p.-rings by Goodearl [5, Theorem 1.1], and $\pi$-regular rings are generalized p.p.-rings.

In [2] Birkenmeier et al. introduced the concept of principally quasi-Baer rings. A ring $R$ is right (left) principally quasi-Baer if the right (left) annihilator of a principal right (left) ideal of $R$ is generated by an idempotent. In [9] Moussavi et al. initiated

Key words and phrases. Generalized quasi-Baer rings, Ore extensions, Annihilator.

2010 Mathematics Subject Classification. 16S36, 16D25.

Received: December 9, 2012

Accepted: November 17, 2014. 
the study of generalized right (principally) quasi-Baer rings. A ring $R$ is generalized right (principally) quasi-Baer if for any (principal) right ideal $I$ of $R$, the right annihilator of $I^{n}$ is generated by an idempotent for some positive integer $n$, depending on $I$.

In [3, Theorem 1.2] Birkenmeier et al. showed that if a ring $R$ is quasi-Baer then $R[x ; \sigma]$ is a quasi-Baer ring when $\sigma$ is an automorphism of $R$. To study quasi-Baerness (right principally quasi-Baerness) of Ore extensions of a quasi-Baer (right principally quasi-Baer) ring, in [7] Hong et al. proved that $R[x ; \sigma ; \delta]$ over a quasi-Baer ring $R$ is a quasi-Baer ring when $\sigma$ is an automorphism of $R$. In this paper we prove that $R[x ; \sigma ; \delta]$ over a generalized quasi-Baer ring is a generalized quasi-Baer ring.

\section{Main Results}

Given a ring $R$, right annihilator of $I$ in $R$ is $r_{R}(I)=\{a \in R \mid I a=0\}$. Left annihilator is defined analogously. The Ore extension $R[x ; \sigma ; \delta]$ is the polynomial ring over $\mathrm{R}$, subject to $x r=\sigma(r) x+\delta(r)$ for any $r \in R$. Note that $R[x ; \sigma ; \delta]$ is written by $R[x ; \sigma]$ and $R[x ; \delta]$ when $\delta=0$ and $\sigma$ is the identity map, respectively. Following [7], if $R$ is a semiprime ring with $\sigma(I) \subseteq I$ for any ideal $I$ of $R$ then $R$ is a quasi-Baer ring if and only if $R[x ; \sigma ; \delta]$ is a quasi-Baer ring. By same method we prove following theorems.

Theorem 2.1. If $R$ is a generalized quasi-Baer ring, then $R[x ; \sigma ; \delta]$ is a generalized quasi-Baer ring.

Proof. Let $I$ be an ideal of $R[x ; \sigma ; \delta]$ and $J=\left\{a \in R \mid a x^{n}+\right.$ terms of lower degree $\in$ $I$ for some $0 \leq n \in \mathbb{Z}\}$. Then $J$ is an ideal of $R$. Note that $\sigma(J) \subseteq J$, and so $J \subseteq \sigma^{-1}(J)$. Let $\dot{J}=\sum_{k \geq 1} \sigma^{-k}(J)$. Then $\dot{J}$ is also an ideal of $R$ and $J \subseteq J^{\prime}$. Moreover, $\sigma(\dot{J})=\dot{J}=\sigma^{-1}(\dot{J})$. If $d \in \dot{J}, d=\sigma^{-k_{1}}\left(d_{1}\right)+\cdots+\sigma^{-k_{i}}\left(d_{i}\right)$, where $d_{k} \in J$ for $k \in\{1, \ldots, i\}$. Since $\sigma(J) \subseteq J, \sigma(d)=\sigma\left(\sigma^{-k_{1}}\left(d_{1}\right)\right)+\cdots+\sigma\left(\sigma^{-k_{i}}\left(d_{i}\right)\right)=\sigma^{-k_{1}}\left(\sigma\left(d_{1}\right)\right)+$ $\cdots+\sigma^{-k_{i}}\left(\sigma\left(d_{i}\right)\right) \in \dot{J}^{\prime}$. Thus $\sigma(\dot{J}) \subseteq J^{\prime}$ and $J^{\prime} \subseteq \sigma^{-1}(\dot{J})$, obtaning from the difinition of $\dot{J}, \sigma^{-1}(\dot{J}) \subseteq \dot{J}$. Hence, $\sigma^{-1}(\dot{J})=J^{\prime}$ and so $\sigma(\dot{J})=\dot{J}$, obtaning $\sigma^{u}(\dot{J})=\dot{J}$ for any integer $u$. By assumption, $r_{R}\left(J^{n}\right)=e R$ for some $e^{2}=e \in R$ and so $\dot{J}^{n} e=0$. We will show that $r_{R[x ; \sigma ; \delta]}\left(I^{n}\right)=e R[x ; \sigma ; \delta]$. Let $g(x)=b_{0}+b_{1} x+\cdots+b_{n} x^{n} \in R[x ; \sigma ; \delta]$ and $f(x) g(x)=0$ for any $f(x)=a_{0}+a_{1} x+\cdots+a_{m} x^{m} \in I^{n}$. Note that for any $r \in R$,

$$
\begin{aligned}
r x^{i}= & x^{i} \sigma^{-i}(r)-\left(\sum_{s+t=i-1} \sigma^{s} \delta \sigma^{t}\left(\sigma^{-i}(r)\right)\right) x^{i-1} \\
& -\cdots-\left(\sum_{s+t=i-1} \delta^{s} \sigma \delta^{t}\left(\sigma^{-i}(r)\right)\right)-\delta^{i}\left(\sigma^{-i}(r)\right) .
\end{aligned}
$$

We can rewrite $f(x)=c_{0}+x c_{1}+\cdots+x^{m} c_{m}$, where $c_{m}=\prod_{i=1}^{n} \sigma^{\sum_{k=i}^{n}\left(-m_{k}\right)}\left(a_{m_{i}}\right) \in \dot{J}^{n}$.

Thus we have the following

$$
\left(c_{0}+x c_{1}+\cdots+x^{m} c_{m}\right)\left(b_{0}+b_{1} x+\cdots+b_{n} x^{n}\right)=0 .
$$


Claim 2.1. $J^{n} b_{n}=0$ and $b_{n}=e b_{n}$.

From equation (2.1), we have $c_{m} b_{n}=0$. Since

$$
\begin{aligned}
& c_{m}=\prod_{i=1}^{n} \sigma^{\sum_{k=i}^{n}\left(-m_{k}\right)}\left(a_{m_{i}}\right) \in J^{n}, \\
& \prod_{i=1}^{n} \sigma^{\sum_{k=i}^{n}\left(-m_{k}\right)}\left(a_{m_{i}}\right)\left(b_{n}\right) \in J^{n} b_{n},
\end{aligned}
$$

we have $a_{m_{1}} \sigma^{m_{1}}\left(a_{m_{2}}\right) \sigma^{m_{1}+m_{2}} \cdots\left(a_{m_{m-1}}\right) \sigma^{m_{1}+m_{2}+\cdots+m_{m-1}}\left(a_{m_{m}}\right) \sigma^{m_{1}+m_{2}+\cdots+m_{m}}\left(b_{n}\right)=0$. Then for any integer $k \geq 0, \sigma^{-k}\left(a_{m_{1}}\right) \sigma^{-k+m_{1}}\left(a_{m_{2}}\right)+\cdots+\sigma^{-k+m_{1}+\cdots+m_{m}}\left(b_{n}\right)=0$. Thus $\bar{J}^{n} \sigma^{-k+m_{1}+c+m_{m}}\left(b_{n}\right)=0$. Since $\sigma^{k-m_{1}-\cdots-m_{m}}\left(\dot{J}^{n}\right)=\dot{J}^{n}$ then $\dot{J}^{n} b_{n}=0$. Hence $b_{n} \in r_{R}\left(\dot{J}^{n}\right)=e R$, and therefore $b_{n}=e b_{n}$.

Claim 2.2. $c_{i} e=0$ for any $0 \leq i \leq m$.

Since $c_{m} \in J^{n}, f(x) e=\left(c_{0}+x c_{1}+\cdots+x^{m} c_{m}\right) e=c_{0} e+x c_{1} e+\cdots+x^{m-1} c_{m-1} e \in I^{n}$. Then $\sigma^{m-1}\left(c_{m-1} e\right) \in J^{n} \subseteq J^{n}$, and so $c_{m-1} e \in \sigma^{-(m-1)}\left(\dot{J}^{n}\right) \subseteq \dot{J}^{n}$. Hence, $c_{m-1} e=$ $\left(c_{m-1} e\right) e \in J^{n} e=0$. Continuing this process, We have $c_{i} e=0$ for any $0 \leq i \leq m$.

Claim 2.3. $g(x) \in e R[x ; \sigma ; \delta]$.

From Claims 2.1 and 2.2, we have

$$
\begin{aligned}
0 & =f(x) g(x) \\
& =x^{m} c_{m} b_{n} x^{n}+\left(x^{m} c_{m} b_{n-1} x^{n-1}+x^{m-1} c_{m-1} b_{n} x^{n}\right)+\cdots+c_{0} b_{0} \\
& =\left(x^{m} c_{m} b_{n-1} x^{n-1}+x^{m-1} c_{m-1} e b_{n} x^{n}\right)+\cdots+c_{0} b_{0} \\
& =x^{m} c_{m} b_{n-1} x^{n-1}+\cdots+c_{0} b_{0} .
\end{aligned}
$$

So, $\sigma^{m}\left(c_{m} b_{m-1}\right)=0$ and we have that $c_{m} b_{n-1}=0$. Then by Claim 2.1, $\dot{J}^{n} b_{n-1}=0$, and it follows that $b_{n-1}=e b_{n-1}$. Then equation (2.2) becomes

$$
\begin{aligned}
0 & =\left(x^{m} c_{m} b_{n-2} x^{n-2}+x^{m-1} c_{m-1} b_{n-1} x^{n-1}+x^{m-2} c_{m-2} b_{n} x^{n}\right)+\cdots+c_{0} b_{0} \\
& =\left(x^{m} c_{m} b_{n-2} x^{n-2}+x^{m-1} c_{m-1} e b_{n-1} x^{n-1}+x^{m-2} c_{m-2} e b_{n} x^{n}\right)+\cdots+c_{0} b_{0} \\
& =x^{m} c_{m} b_{n-2} x^{n-2}+\cdots+c_{0} b_{0} .
\end{aligned}
$$

Thus $c_{m} b_{n-2}=0$, and so $J^{n} b_{n-2}=0$. Hence, we also have $b_{n-2}=e b_{n-2}$. Continuing this process, we have $b_{j}=e b_{j}$ for any $0 \leq j \leq n$. Consequently, we have $g(x)=$ $b_{0}+b_{1} x+\cdots+b_{n} x^{n}=e b_{0}+e b_{1} x+\cdots+e b_{n} x^{n} \in e R[x ; \sigma ; \delta]$.

Conversely, let $f(x)=c_{0}+x c_{1}+\cdots+x^{m} c_{m} \in I^{n}$. Then by Claim 2.2, $f(x) e=0$. Thus $I^{n} e=0$, and so $e R[x ; \sigma ; \delta] \subseteq r_{R[x ; \sigma ; \delta]}\left(I^{n}\right)$. Consequently, $R[x ; \sigma ; \delta]$ is a generalized quasi-Baer ring.

In the following proposition we see that the converse of Theorem 2.1 is correct when $R$ be a semiprime ring. 
Proposition 2.1. Let $R$ be a semiprime ring with $\sigma(I) \subseteq I$ for any ideal $I$ of $R$. If $R[x ; \sigma ; \delta]$ is a generalized quasi-Baer ring, then $R$ is a generalized quasi-Baer ring.

Proof. Let $J$ be an ideal of $R$. Since $R[x ; \sigma ; \delta]$ is a generalized quasi-Baer ring, then $r_{R[x ; \sigma ; \delta]}\left((J R[x ; \sigma ; \delta])^{n}\right)=e(x) R[x ; \sigma ; \delta]$ for $e(x)^{2}=e(x)=e_{0}+e_{1} x+\cdots+$ $e_{t} x^{t} \in R[x ; \sigma ; \delta]$ and a positive integer $n$. Since $e(x) R[x ; \sigma ; \delta]$ is an ideal, $(e(x)-$ 1) $R[x ; \sigma ; \delta] e(x)=0$. Hence we have $e_{t} R \sigma^{t}\left(e_{t}\right)=0$. Since $R$ is semiprime, $\sigma^{t}\left(e_{t}\right) R e_{t}=$ 0 . Then $\sigma^{t}\left(e_{t}\right) R \sigma^{t}\left(e_{t}\right) \subseteq \sigma^{t}\left(e_{t}\right) R \sigma^{t}\left(R e_{t} R\right) \subseteq \sigma^{t}\left(e_{t}\right) R e_{t} R=0$ and so $\sigma^{t}\left(e_{t}\right) R \sigma^{t}\left(e_{t}\right)=0$. Since $\sigma$ is an automorphism of $R$ and $R$ is semiprime, $e_{t}=0$. Continuing this process, we have $e(x)=e_{0}$, where $e_{0}^{2}=e_{0} \in R$. Thus $r_{R[x ; \sigma ; \delta]}\left((J R[x ; \sigma ; \delta])^{n}\right)=e_{0} R[x ; \sigma ; \delta]$. We claim that $r_{R}\left(J^{n}\right)=e_{0} R$. Obviously $e_{0} R \subseteq r_{R}\left(J^{n}\right)$. If $b \in r_{R}\left(J^{n}\right)$ then $J^{n} b=0$. Since $\sigma^{s}(b) \in R b R, J^{n} \sigma^{s}(b) \subseteq J^{n} b R=0$ and so $J^{n} \sigma^{s}(b)=0$ for any integer $s \geq 0$. Then by [7, Lemma 9], we have $J^{n} \sigma^{m_{1}} \delta^{n_{1}} \cdots \sigma^{m_{u}} \delta^{n_{u}}(b)=0$ for any integers $m_{v}, n_{v} \geq 0$. Thus $J^{n} R[x ; \sigma ; \delta] b=0$ and so $b \in r_{R[x ; \sigma ; \delta]}\left((J R[x ; \sigma ; \delta])^{n}\right)=e_{0} R$. Hence, $r_{R}\left(J^{n}\right) \subseteq e_{0} R$. Consequently, $R$ is a generalized quasi-Baer ring.

Corollary 2.1. Let $R$ be a semiprime ring with $\sigma(I) \subseteq I$ for any ideal $I$ of $R$. Then $R[x ; \sigma ; \delta]$ is a generalized quasi Baer ring if and only if $R[x ; \sigma ; \delta]$ is a quasi-Baer ring.

Proof. If $R[x ; \sigma ; \delta]$ is a generalized quasi Baer ring then $R$ is a generalized quasi-Baer ring. Since $R$ is a semiprime ring by [9, Proposition 2.2], $R$ is a quasi-Baer ring. Then by $[7$, theorem 1$], R[x ; \sigma ; \delta]$ is a quasi-Baer ring. The converse is clear.

Acknowledgment: The authors wish to express their gratitude to the referee for his/her helpful suggestions and comments for the improvement of the paper.

\section{REFERENCES}

[1] S. K. Berberian, Baer * rings, Grundlehren Math. Wiss. Band 195; Springer: Berlin, 1972.

[2] G. F. Birkenmeier, J. Y. Kim, J. K. Park, Principally quasi-Baer rings, Communication in Algebra 29(2) (2001) 639-660.

[3] G. F. Birkenmeier, J. Y. Kim, J. K. Park, Polynomial extensions of Baer and quasi-Baer rings, J. Pure Appl. Algebra 159 (2001) 25-42.

[4] W. E. Clark, Twisted matrix units semigroup algebras, Duke Math. J. 34 (1967) 417-424.

[5] K. R. Goodearl, von Neumann regular rings, Krieger: Malabar, 1991.

[6] C. Huh, H. K. Kim, Y. Lee, p.p.-rings and generalized p.p.-rings, J. Pure Appl. Algebra 167 (2002a) 37-52.

[7] C. Y. Hong, N. K. Kim, Y. Lee, Ore extensions of quasi-Baer rings, Communication in Algebra 37 (2009) 2030-2039.

[8] I. Kaplansky, Rings of Operators, Benjamin, New York, 1965.

[9] A. Moussavi, H. Haj Seyyed Javadi, E. Hashemi, Generalized quasi-Baer rings, Communication in Algebra 33 (2005) 2115-2129.

[10] C. E. Ricart, Banach algebras with an adjoint operation, Ann. of Math. 47 (1946) 528-550.

${ }^{1}$ Shahed Univesity, TEHRAn, IRAN

E-mail address: anzani@shahed.ac.ir

E-mail address: h.s.javadi@shahed.ac.ir 